\title{
Use of medical informatics for management of multiple sclerosis using a chronic-care model
}

\author{
Michael J. Hatzakis Jr, MD; ${ }^{1-3 *}$ Craig Allen, MS; ${ }^{1,4}$ Mark Haselkorn, PhD; ${ }^{1,4}$ Stephen M. Anderson, MS; ${ }^{1-2}$ \\ Paul Nichol, MD; ${ }^{1-2,4}$ Charles Lai, MS; ${ }^{1,4}$ Jodie K. Haselkorn, MD, MPH ${ }^{1-3,5}$ \\ ${ }^{1}$ Multiple Sclerosis Center of Excellence West, Veterans Health Administration, Seattle, WA; ${ }^{2}$ Department of Veterans \\ Affairs Puget Sound Health Care System, Seattle, WA; Departments of ${ }^{3}$ Rehabilitation Medicine, ${ }^{4}$ Internal Medicine, \\ ${ }^{5}$ Epidemiology, University of Washington, Seattle, WA
}

\begin{abstract}
The mission of the Multiple Sclerosis Centers of Excellence (MSCoEs) is to optimize the services veterans with multiple sclerosis (MS) receive across the U.S. Veterans Health Administration. To accomplish this mission, the MSCoE West has adopted a collaborative chronic-disease management strategy along the lines of the model described by Wagner and colleagues. This model describes an organized, integrated, proactive, and population-based approach to patient care that includes healthcare delivery system change and patient-based self-management. While Wagner's model is described independent of information technology, the majority of actions called for in that model benefit tremendously from the application of a powerful and well-integrated informatics infrastructure designed to serve and support populations with chronic disease. Key elements such as goals and actions encourage high-quality care for those with chronic illnesses.
\end{abstract}

Key words: chronic disease, computerized order entry, computerized physician order entry, delivery of healthcare, health services, medical informatics, multiple sclerosis, physician's practice patterns, practice guidelines, veterans.

\section{INTRODUCTION}

The mission of the Multiple Sclerosis Centers of Excellence (MSCoEs) is to optimize the services that veterans with multiple sclerosis (MS) receive across the U.S. Veterans Health Administration (VHA). To accomplish this mission, the MSCoE West is implementing a model of care adapted from other chronic-care models that have been successful in managing large populations with chronic diseases such as diabetes and asthma [1-2]. Wagner and colleagues have described a system approach to the care of populations with chronic disease that is built around two essential elements: (1) "prepared, proactive, practice teams" that use tools such as evidence-based guidelines, data repositories, and an electronic medical record (EMR) to manage high-risk groups with specific conditions and (2) "an educated and empowered patient" who receives education and training

\footnotetext{
Abbreviations: $\mathrm{CCR}=$ computerized clinical reminders, $\mathrm{CDR}=$ clinical data repository, CPRS = Computerized Patient Record System, CSF = cerebrospinal fluid, DMA = diseasemodifying agent, EMR = electronic medical record, FAQ = frequently asked questions, ICD = International Classification of Diseases, IOM = Institute of Medicine, IT = information technology, MRI = magnetic resonance imaging, $\mathrm{MS}=$ multiple sclerosis, MSCoE = MS Center of Excellence, QI = quality improvement, VA = Department of Veterans Affairs, VHA = Veterans Health Administration, VISN = Veterans Integrated Service Network.

*Address all correspondence to Michael J. Hatzakis Jr, MD; VA Puget Sound Health Care System, Seattle Division, Rehabilitation Care Services, S-117-RCS, 1660 South Columbian Way, Seattle, WA 98108; 206-277-1792; fax: 206-764-2263. Email: mail@hatzakis.net

DOI: $10.1682 / J R R D .2004 .10 .0135$
} 
and is an active participant in his or her healthcare [3-4]. This organizational approach is built to support planned, productive interactions with the goal of optimizing desired outcomes for a particular population. Key elements such as goals and actions encourage high-quality care for those with chronic illnesses (Figure 1). In addition, an Institute of Medicine (IOM) report published in 2002 presents concepts for improving the quality of healthcare and suggests an approach to chronic care that has many of the same basic elements described by Wagner [3]. Given the chronic-care model's effectiveness in chronic illnesses such as diabetes and asthma and its general acceptance in the medical community, we chose to adapt features and apply them to the delivery of services to veterans with MS in the VHA.

Many chronic-care models have been developed without computerization; however, when one reviews the components of a well-integrated health information system, it becomes apparent that such an infrastructure is at least highly desired for most of these actions and nearly essential for many. For a nationwide comprehensive system like the VHA, promoting effective improvement strategies aimed at comprehensive system change and identifying relevant subpopulations for proactive care may be impossible without a sophisticated clinical data repository (CDR) of all interactions within a healthcare system. Similarly, organizing internal and community resources to provide ongoing self-management support to patients and sharing evidence-based guidelines and information with patients to encourage their participation may be also impossible without taking advantage of the VHA's widely used Web site to integrate existing resources and guidance and effectively link veterans to that information. Even for systems with a relatively small number of patients, research has shown that the use of informatics infrastructure can improve outcomes [5]. The MSCoEs are fortunate that the VHA has a rich informatics infrastructure that readily supports the implementation of a chronic-care model.

This article explores how the MSCoEs are developing and employing an informatics infrastructure in support of a chronic-care management strategy that optimizes services to the national population of veterans with MS. In particular, we focus on four informatics elements that leverage the VHA's existing powerful infrastructure: (1) a CDR, (2) the World Wide Web, (3) an enhanced EMR system, and (4) telehealth delivery of services.

\section{MULTIPLE SCLEROSIS CLINICAL DATA REPOSITORY}

"Information about patients, their care, and their outcomes is an essential ingredient of all population-based strategies to improve chronic illness care" [4]. A CDR or "chronic disease registry" (as Wagner calls it) provides the clinician with the information he or she needs to shift from an "episodes of care" model to one that considers and anticipates the needs of populations with similar features, i.e., MS and diabetes. Needs, interventions, and outcome evaluations are assessed with the use of population data. Such information drives proactive care that empowers providers to anticipate the needs of patients.

A key mission of the MSCoE is to "develop large databases to support clinical care and research to allow better understanding of the nation's MS population" [6]. To achieve this goal, the MSCoEs have developed an MS CDR using multiple dimensions of data organized in a relational format. The MS CDR is a collection of health information that has been extracted from national data stores of regional EMR systems [7]. Data such as outpatient visits, inpatients stays, surgical procedures, laboratory results, prescriptions, and expenditures are combined in a large relational data structure designed to easily access and to support analysis across the multiple types of data. The MSCoE relational structure allows multiple related data tables with varying table structures to be combined in one data structure such as Medicare data, national death registry lists, magnetic resonance imaging (MRI) results, or banks of DNA (deoxyribonucleic acid) data.

Many registries have been described as paper-based lists of patients meeting a set of inclusion criteria added manually as they were seen and evaluated [5,8-9]. Providers used these paper-based registries to assimilate essential information used to identify needs and to stratify patient subpopulations. Using paper or electronic lists to keep track of individuals meeting given criteria limits the ability of providers to use this information to manage patients as populations. For example, a diabetes registry consisting of individuals meeting certain criteria cannot be retrospectively segregated into subpopulations unless the segregation variable has been collected in the proper format. In addition, the process of creating feedback reports and routine quality improvement (QI) summaries from early paper or electronic registries is extremely labor-intensive, because it requires an individual to manually review charts to determine whether they meet certain quality measures. Health information technology (IT) has 
(1) The Community: Mobilize community resources to meet needs of patients.

Encourage patients to participate in effective community programs.

Form partnerships with community organizations to support and develop interventions that fill gaps in needed services.

Advocate for policies to improve patient care.

(2) The Health System: Create a culture, organization and mechanisms that promote safe, high-quality care.

Visibly support improvement at all levels of the organization, beginning with the senior leader.

Promote effective improvement strategies aimed at comprehensive system change.

Encourage open and systematic handling of errors and quality problems to improve care.

Provide incentives based on quality of care.

Develop agreements that facilitate care coordination within and across organizations.

(3) Self-Management Support: Empower and prepare patients to manage their health and healthcare.

Emphasize the patient's central role in managing their health.

Use effective self-management support strategies that include assessment, goal-setting, action planning, problem-solving, and follow-up.

Organize internal and community resources to provide ongoing self-management support to patients.

(4) Delivery System Design: Assure the delivery of effective, efficient clinical care, and self-management support.

Define roles and distribute tasks among team members.

Use planned interactions to support evidence-based care.

Provide clinical case management services for complex patients.

Ensure regular follow-up by the care team.

Give care that patients understand and that fits with their cultural background.

(5) Decision Support: Promote clinical care that is consistent with scientific evidence and patient preferences.

Embed evidence-based guidelines into daily clinical practice.

Share evidence-based guidelines and information with patients to encourage their participation.

Use proven provider education methods.

Integrate specialist expertise and primary care.

(6) Clinical Information Systems: Organize patient and population data to facilitate efficient and effective care.

Provide timely reminders for providers and patients.

Identify relevant subpopulations for proactive care.

Facilitate individual patient care planning.

Share information with patients and providers to coordinate care.

Monitor performance of practice team and care system.

\section{Figure 1.}

Elements, goals, and actions of Wagner's chronic-care model (Improving Chronic Illness Care [homepage on the Internet]. Seattle (WA): Improving Chronic Illness Care; c2006 [updated 2006; cited 2005 Apr 21]. Improving Chronic Illness Care, Delivery System Design; [1 screen]. Available from: http://www.improvingchroniccare.org/change/model/deliv_design.html).

matured from paper-based systems or individual electronic records (akin to word processor documents) to organized systems that collect and store multiple types of healthcare data that enable providers and researchers to ask a variety of questions of large populations. These large resulting databases are referred to as CDRs. The terms "repository" and "registry" are often used interchangeably. A registry is commonly considered a list of individuals as they pass through a medical system. A registry is populated as individuals are identified as having a given characteristic. Other data may be added during this process as well. A CDR is generally a "store" of all patient data for data relating to their interactions in a healthcare system. IT specialists often refer to this as a "datamart" or a collection of transactions, patient data, prescriptions, etc, for administrative and other generalpurpose use. The size and members of a disease-specific data repository may be fluid as an algorithm is refined or as cases are validated and those miscoded are removed. Chronic-care data repositories, such as the MS CDR, extract comprehensive data from a subset of the VHA's EMR. The form data stored in the EMR is not easily accessible. In the case of the VHA's EMR, records are stored one patient at a time, such that questions that query data across patients are very difficult. EMR data provide a rich source of cases with a wide variety of data types 
covering all aspects of chronic care, from diagnoses and treatments to outcomes and costs. This is consistent with IOM recommendations for an optimal system approach to chronic disease as well as the previously described chronic-care models. Use of a CDR is widely supported in the literature and clinical experience. The IOM report on Fostering Rapid Advances in Health Care [10] states, "ideal information and communications technology support include the use of . . . registries." In fact, the advantages of using disease registries were recognized long before the development of computer databases [9,11-12].

The population-based chronic-care model focuses on "evidence-based, planned care," and a comprehensive chronic-care data repository can be central to providing such care. In fact, an electronic patient registry can improve the effectiveness of all six chronic-care model domains described in Figure 1. A disease repository can "organize patient and population data to facilitate efficient and effective care," offer "ready access to meaningful data drives QI efforts," and "create a culture, organization and mechanisms that promote safe, highquality care" and thereby "assures the delivery of effective, efficient clinical care" [3-4]. Such data provide feedback to individual practitioners in an effort to stimulate individual practitioner growth, allow meaningful benchmarking with colleagues, and identify systematic problems in care processes $[4,13]$. The feedback can be delivered as reports or in clinical reminders that are integrated into the treatment setting. An effective CDR has many characteristics that allow it to be more effective and have greater effect than the use of simple registries or lists of patients. A well-designed CDR can easily define and analyze subgroups of a greater population. Adding data with new dimensions does not affect the structure of a data repository; for example, data stored in the domain of time can be mixed with data in the domain of individual patients or as individual contacts. This structure prevents future limitations of added data. The ability to create subpopulations easily allows views from various perspectives, depending on the needs of the viewer. This constant interaction with such a repository in routine clinical care also helps to maintain maximal data quality and integrity.

\section{POPULATION IDENTIFICATION}

To take advantage of rich sources of data such as the MS CDR, one must use algorithms to identify individuals from the health system's EMR database. This algorithm must be carefully designed and tested to correctly classify and separate the individuals who truly have MS from those who do not. Concepts such as sensitivity, specificity, and validity are important. Sensitivity, in this context, is the ability of the selection algorithm to identify those who have been given a diagnosis of MS. Sensitivity, in this case, is the number of individuals identified divided by the individuals who have clinical evidence of MS based on a "gold standard" process, such as patient examinations or detailed chart review. In this case, we used an adaptation of the McDonald et al. criteria [14] (Figure 2). An algorithm with a high sensitivity will identify a maximal number of individuals with MS, at the expense of falsely identifying others without MS. In the MSCoE repository, the International Classification of Diseases (ICD) code corresponding to MS (340) identifies patients from the Department of Veterans Affairs (VA's) data repository. However, the selection algorithm is limited to the quality of the underlying data. In QI reviews, many individuals did not have a diagnostic code when they actually had MS. We added other criteria to increase the sensitivity of the algorithm. We were aware that a number of veterans may be receiving disease-modifying agents (DMAs), but they were not coded as having MS. Given that few, if any, individuals are given this medication for other disease besides MS, specificity of the algorithm would not be reduced. To improve sensitivity, we identified individuals who are either receiving or have received a DMA in the past. One could also evaluate other clinical characteristics, such as the presence of oligoclonal bands in the cerebrospinal fluid (CSF) or MRI findings, to determine if they enhance the identification of individuals with electronic evidence for MS, but we did not use these characteristics because of technical constraints and the probability that specificity would be reduced. The final case identification algorithm for MS is:

- INPATIENT ICD = 340, OR

- OUTPATIENT ICD = 340, OR

- PRESENCE OF PREVIOUS RX FOR DMA, OR

- ACTIVE RX FOR DMA (Interferon $\beta$-1a, Interferon $\beta$-1b, OR glatiramer acetate).

Specificity, in this setting, is defined as the number of individuals who have no electronic evidence of MS and who are not in the registry, divided by the number of individuals who do not have MS. Specificity is lowered because of false positives. False positives include individuals who were given an ICD-9 Code 340 for an evaluation 


\begin{tabular}{|c|c|}
\hline $\begin{array}{l}\text { Has Disease } \\
\text { Present in } \\
\text { Registry }\end{array}$ & $\begin{array}{c}\text { Does Not Have } \\
\text { Disease Present } \\
\text { in Registry }\end{array}$ \\
\hline TRUE POSITIVE & FALSE POSITIVE \\
\hline [a] & [b] \\
\hline $\begin{array}{l}\text { Has Disease } \\
\text { Not Present } \\
\text { in Registry }\end{array}$ & $\begin{array}{c}\text { Does Not Have } \\
\text { Disease Not Present } \\
\text { in Registry }\end{array}$ \\
\hline FALSE NEGATIVE & TRUE NEGATIVE \\
\hline [c] & [d] \\
\hline \multicolumn{2}{|c|}{ Sensitivity $=\frac{a}{a+c}$ Specifity $=\frac{d}{a+b}$ Positive Predictive Value $=\frac{a}{a+b}$} \\
\hline
\end{tabular}

Figure 2.

Sensitivity and specificity.

in which MS was "ruled out," for individuals who have "possible MS," and for whom the code was entered in error. The validation process consists of chart review and application of the McDonald et al. criteria to determine the validity of the diagnosis of MS [14]. Ongoing efforts to accurately and validly classify individuals with MS are an important part of a data repository.

\section{FLEXIBILITY}

In addition to developing an effective algorithm and inclusion criteria, one must also ensure that the MS CDR is flexible and can accommodate novel types of data. In this way, new data from outside sources (e.g., Medicare) or data enriched by data collection activities (e.g., through surveys, reminders, or templates) may be added. The relational data repository architecture must be able to support future added data both in depth (more data for same population) and breadth (new types of data describing the population).

The system architecture on which a chronic disease health data repository is based must also support rapid development of information products and must support a variety of information activities, including data exploration, analysis, and reporting services. These activities relate directly to many of the requirements to care for individuals with chronic disease. However, these products can be quite disparate in terms of optimal data structures, presenting challenges to data management and system design. For example, to determine which patients are the highest 10 percent of users of services, one might require access to data at a more detailed level, with which to segregate populations of individuals. Monitoring of facility performance against certain measures might require the creation of high-level, precalculated reports. Statistical analyses often require data structures that are "flattened" into wide tables, perhaps putting multiple occurrences of an event (e.g., outpatient visit) on the same row, while the delivery of Web reports to providers, administrators, and the public would require summarized tables.

\section{DATA QUALITY AND INTEGRITY}

Efforts in QI draw heavily from data repositories. A well-designed CDR increases the number and quality of tools available to healthcare providers, giving them more ways to see the impact of current care practices on the population affected by chronic disease. With the ability to see and use data in more ways, providers not only obtain better feedback on the healthcare system performance but also develop a better sense of the quality of the data and their data needs. In our experience, this direct use of the data by providers has helped identify errors in the database and has led to innovative processes to help correct erroneous data using provider input. Involving providers in multiple entry points of clinical information helps create a system that is provider friendly and maximizes provider "buy-in." This leads to improved quality of data from the feeder systems and promotes a culture well versed in data analysis and QI [13,15]. A well-designed data repository is easily accessible by multiple key providers or administrators. These individuals improve input of data as the repository is used more frequently to change clinical behavior based on an opportunity to evaluate their performance over time. Performance can be fed back to providers with through the use of aggregate reports or in the form of patient-specific reminders. An example of one of the VHA reminders is, "This patient needs a flu vaccine." 
The quality and acceptability of feedback given to providers depend on the error rate and timeliness of the information driving them [16-17]. For MSCoE, accurate and up-to-date information to drive reminders, feedback reports, and patient or physician profiling is available from our growing MS CDR. Our clinicians and administrators have analyzed repository data to better understand the population of approximately 26,000 veterans who were treated for MS between 1998 and 2002 [6]. Given that a disease-specific CDR is drawn from a hospital's CDR with an algorithm, that data must be drawn regularly. How frequently the data are drawn (e.g., yearly, quarterly, or monthly) will drive long-term planning and shorter-term management strategies. More frequently refreshing the disease-specific data repository allows prompt feedback to clinical and administrative personnel but is highly effortintensive. Less-frequent refreshes, say, annually, are easier to perform but cause a 1-year delay before feedback about clinical changes can be provided. MSCoE is starting with an annual refresh schedule and increasing as refresh processes become routine and demand grows. A more frequent refresh schedule will encourage increased usage and will likely improve accuracy.

\section{INFORMATION ACCESS}

Information derived from registries like MSCoE's MS CDR can be used in myriad ways. For the MSCoE, it is the first time that we are able to identify a population of veterans with MS and characterize them so we can deliver high-quality, consistent services; begin to meet unmet needs; and plan for future needs. For large healthcare organizations like the VHA, the information can drive critical business decisions ranging from how to address the needs of patients to how to accurately bill third-party payers. Data repositories can be used to segregate subpopulations on the basis of criteria such as laboratory tests, presence of comorbid conditions, and healthcare expenditures. If information from these repositories can be made easily accessible to veterans, then they can advocate for their own care. Individuals may remind their clinicians to prescribe important medications, conduct routine screening tests, and attempt interventions such as vaccinations when they are appropriate. Future systems may support a model where performance data are made available to veterans so they can choose providers or facilities with higher performance ratings.
Given this wide range of potential benefits, design and construction of CDRs have taken on a great deal of importance and many organizations, including the VHA, are investing resources in the creation of reliable, broadly applicable data repositories [7]. The appropriateness and effectiveness of feedback provided to clinicians and patients depend, to a large extent, on the accuracy and completeness of the data in repositories [17]. Erroneous or absent data will have a substantial impact on physician use of decision support systems [16]. Ongoing efforts to enhance the data accuracy, validity, and access are keys to a successful repository.

\section{MULTIPLE SCLEROSIS CENTER OF EXCELLENCE WEB SITE}

In response to its mission of "providing a national program of MS education for VHA healthcare providers, veterans, their families, and caregivers” [6], the MSCoE established an informational web site. The MSCoE Web site has become a primary tool to disseminate knowledge to both providers and veterans with MS. Knowledge has been adapted to audiences who learn in varying styles by providing information in formats such as summaries of "best practices of care" or guidance documents or frequently asked questions (FAQ) that provide knowledge in a "Q\&A" (questions and answers) format. Veterans and providers can ask questions and receive public answers on the Web site. In this way, the MSCoE can both empower veterans with the knowledge they need to drive their own care and prepare providers to care for a population of veterans with specific needs. This mechanism indirectly connects providers and veterans by allowing questions to be asked by veterans and answered by MSCoE staff, all of whom are MS providers.

The MSCoE Web site is an online audience- and community-based MS information portal for providers and veterans with MS. The rationale behind the design of the MSCoE Web site stems from numerous studies that support both a "user-centered design" [18-19] and the use of the Internet to disseminate health information, especially for chronic diseases such as MS [20-21].

In its current version, the MSCoE Web site is organized and divided into sections tailored to the two primary audiences that it serves: healthcare providers and veterans with MS. Both sections contain identical types of content: guidance articles and FAQs aimed at their respective 
audiences and a common library of MS resources. Within these types, all sections are further unified by a common four-part organization of content: (1) Understanding and Diagnosing MS, (2) Treating MS, (3) Managing Health Problems Associated with MS, and (4) Managing Life Issues Associated with MS.

In addition to the organization of content, a Websupported, peer-review process has been developed that supports the development and evaluation of content [22]. This process coordinates a dedicated Web editorial board of VHA MS experts from across the nation, supporting their interactions with internal components of the Web site. Using this process, the board Web site creates, peerreviews, and publishes content for the MSCoEs for the desired audiences.

The MSCoE Web site conducts several functions discussed in the Wagner chronic-care model [3-4]. Specifically, the Web site (1) "share[s] evidenced-based guidelines and information with patients to encourage their participation," (2) "organize[s] internal and community resources to provide ongoing self-management support to patients," and (3) "integrate[s] specialist expertise and primary care."

The first action, sharing peer-reviewed, evidencebased guidance and information with patients, is accomplished with the guidance documents and FAQ board. Evidence of its effectiveness in its first year is seen as increasing feedback - the number of total asked questions-has risen from 27 in August 2004 to over 100 by the end of September 2004. The number of visits has steadily increased from roughly 3,377 in June 2004 to 4,020 in August and 4,250 in September 2004. By July 2004, the number of users visiting multiple Web pages had steadily increased, and by September 2004, this number had stabilized to roughly 200, likely representing a regular, captured audience. The time spent on the site has also stabilized at almost 20 min per visit. This information suggests that more users see the MSCoE Web site each month; the regular users represent roughly 20 percent of all visits to the web site. Veterans can access MSrelated information and even ask questions of MS specialists without traveling to a hospital or getting an appointment at a clinic. The information is oriented toward empowering participation of the individual in his or her own care. In the veteran section, guidance documents are designed to speak to veterans about managing the secondary complications associated with MS and its treatments in their own language, yet the documents are based on peer-reviewed content and expert assessment drawn from the latest evidence-based guidelines. The FAQ section provides veterans with information that can help them take a more active role in their own healthcare, allowing them to ask questions and receive authoritative, but personal, answers from MS professionals. Here is an example:

\section{Question:}

Should I get a flu shot if I have MS and I am on an injectable therapy?

Answer:

According to a recent study published in the Archives of Neurology (2003), and one published in Neurology (2001), the flu vaccine (injectable) is not associated with an increased risk of an MS exacerbation. However, Dr. Dennis Bourdette, Professor of Neurology at Oregon Health \& Science University, has stated that the 2003 nasal spray flu vaccine should not be given to persons with MS. He recommends the injectable flu vaccine over the nasal spray for patients with MS.

The veteran FAQ feature has developed into an effective means to address the information needs of veterans and providers. The FAQ entries published in April/ May 2004 had 25 FAQ submissions between July 1 and September 1, 2004. Over 1,000 unique viewers visited the FAQ page between January and September 2004. The second action discussed in Wagner's model is the organization of internal and community resources to provide ongoing self-management support to patients with MS. The Web site brings together news items with the latest research and treatment developments and presents this MS information to veterans from a single information source. Content creation is collaborative in nature. MSCoE professionals from differing backgrounds and viewpoints review published content. The process of answering incoming FAQ questions, in particular, fosters direct interaction between MSCoE professionals and veterans and gives MSCoE professionals a sense of topics of general interest to veterans. Information about these topics, many of which deal with self-management, is then disseminated via the Web site as FAQ or guidance entries. The content creation process ensures the effective dissemination of information to support ongoing selfmanagement of veterans with MS.

The third action of a chronic-care model, integration of specialist expertise and primary care, is accomplished 
on the Web site through the presentation of evidencebased guidelines and less formal guidance documents for providers, allowing providers to access specialized knowledge on an as-needed basis. Overall, the providers section of the Web site expands on previous work that suggests that the Internet is a promising medium for dissemination of evidence-base guidelines to providers [3$4,6,23]$. The provider guidance documents on the Web site are short, focused pieces that present the latest evidence-based information on specific MS topics of interest, such as use of DMAs, diagnostic criteria, and associated health conditions, such as fatigue and spasticity. The library section also delivers evidence-based information on these and other topics, but in the form of slides or videos from formal presentations by MS specialists. The provider FAQ section is similar to the veteran's section: providers can actively ask MS questions and receive peer-reviewed answers form MSCoE professionals. All the provider content is peer-reviewed. A recent example is-

\section{Question:}

Would you please give some advice regarding diagnosing Devic's Disease? We have a patient who presented a few years ago with transverse myelitis, 1,000 white blood cells in CSF, who now has optic neuritis with 1 cell and 4 oligoclonal bands. No other neurological symptoms. The optic neuritis occurred a few months postpartum.

Thank you.

\section{Answer:}

Devic's (neuromyelitis optica) is a challenging diagnosis. It is often difficult to differentiate from MS. There are now criteria to assist in making the diagnosis. These criteria are-

Absolute criteria (all required):

1. Optic neuritis.

2. Acute myelitis.

3. No evidence of clinical disease outside of the optic nerve or spinal cord.

Supportive criteria (either one major criterion or two minor criteria):

-Major

1. Negative brain MRI at onset (does not meet Paty radiological criteria for MS).

2. Spinal cord MRI with signal abnormality extending over $>3$ vertebral segments.
3. CSF pleocytosis of $>50$ white blood cells $/ \mathrm{mm}^{3}$ or $>5$ neutrophils $/ \mathrm{mm}^{3}$.

-Minor

1. Bilateral optic neuritis.

2. Severe optic neuritis with fixed VA worse than $20 / 200$ in at least one eye.

3. Severe, fixed, attack-related weakness (MRC grade $<2$ ) in one or more limbs.

Looking at these criteria, your case meets the criteria just defined. There is evidence of optic nerve and spinal cord involvement. One major criterion is also met from the CSF white blood cells of 1,000 at the initial presentation. The main question is whether there is clinical disease outside of the optic nerve or spinal cord. The presence of this clinical disease is partly addressed with MRI. The brain MRI early in the course of Devic's is usually normal or near normal. Abnormalities on the brain MRI suggestive of demyelinating disease argue for MS rather than Devic's. Also, the MRI from the initial episode of myelitis may be helpful. Devic's often has extensive spinal MRI changes, spanning 3 or more bony levels. MS tends to have lesions that span fewer than 3 levels, though there may be several of these smaller lesions spread along the spinal cord. Oligoclonal bands may be seen with either disease (though they are less common in Devic's).

The Web site is organized to meet the clinical needs of both primary and specialty care providers, by organizing around the four categories: (1) Understanding and Diagnosing MS, (2) Treating MS, (3) Managing Life Issues associated with MS, and (4) Managing Health Problems Associated with MS. This clinical organization pattern facilitates thinking about guidance in terms of clinical experience for both providers and patients.

\section{ENHANCED ELECTRONIC MEDICAL RECORD SYSTEM}

One of the aims of the MSCoEs is to "apply proven technologies to enhance veteran and clinician decisionmaking.” [3-4]. Literature describing clinician behavior change suggests that interventions should be available at the time of the provider-patient encounter [24-26]. In the VHA model of care, the best way to achieve this kind of 
intervention is through enhancements of the VHA's Computerized Patient Record System (CPRS). CPRS is used across the entire VA healthcare system and almost exclusively at the point of care. Components of CPRS can be customized, allowing providers or health systems to increase efficiency by tailoring their interfaces to their needs. Customization includes enhancing clinician order sets, adding electronic note templates that guide care, and computerized clinical reminders (CCRs) that remind and guide the care of individual patients. The MSCoE has established several electronic note templates and reminders (Figure 3) and has begun the development of MS disease-specific order sets.

CPRS is the VHA-specific implementation of an EMR system. Over the last decade, EMRs have made a gradual transition from archiving and retrieving medical information to assisting in making medical decisions by providing information in new contexts or adding information about clinical standards of care [13,27]. Today, EMRs have an expanded purpose: "to recall observations, to inform others, to instruct students, to gain knowledge, to monitor performance and to justify interventions" [27]. The use of EMR systems results in more complete documentation that more effectively describes the appropriateness of clinical decisions as compared with paper record keeping [28]. Such systems are also more effective tools for provider behavior change [30]. Current EMRs contain clinical decision support systems, some as simple reminders and others as complex standalone systems that synthesize medical information to suggest diagnostic or therapeutic interventions.

Decision support systems promote the practice of clinical care that is consistent with particular guidelines of care, as well as the preferences of the individual patient.

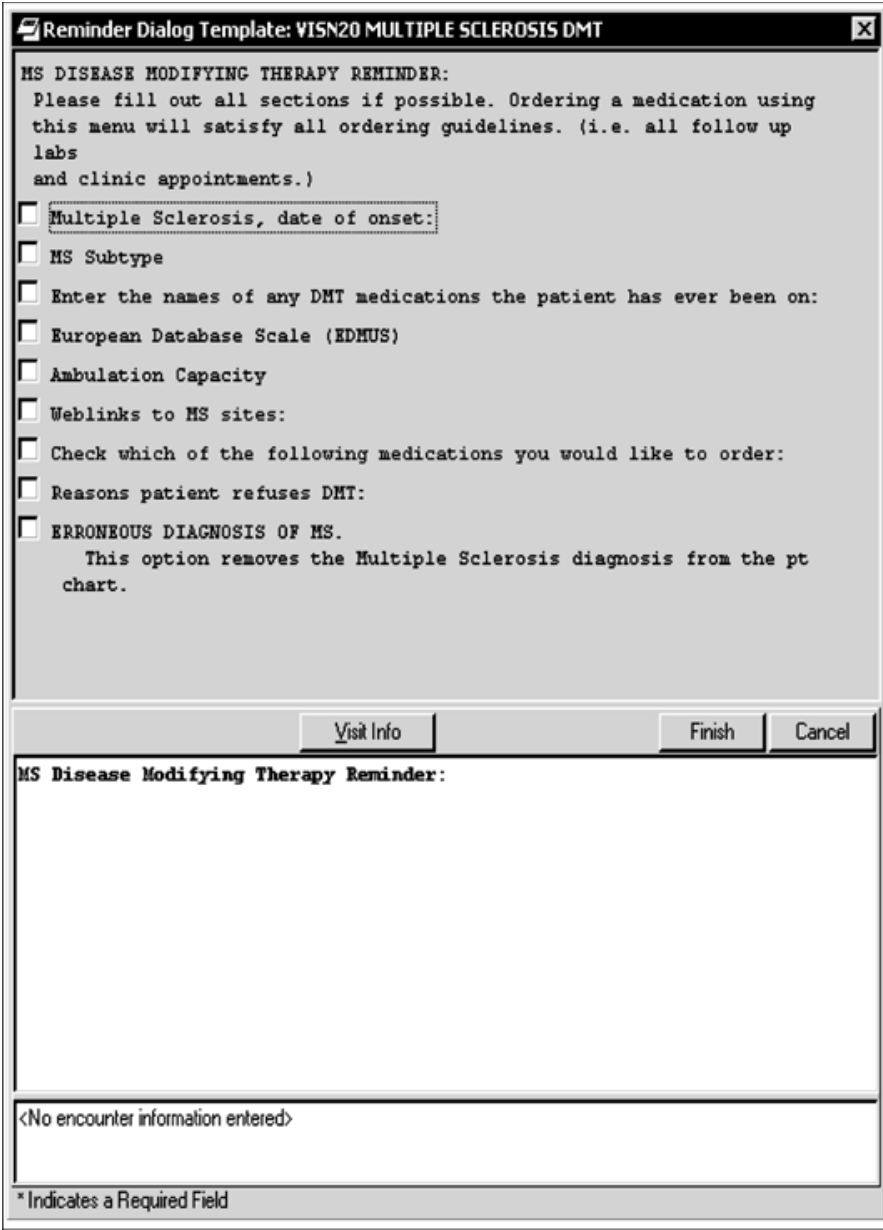

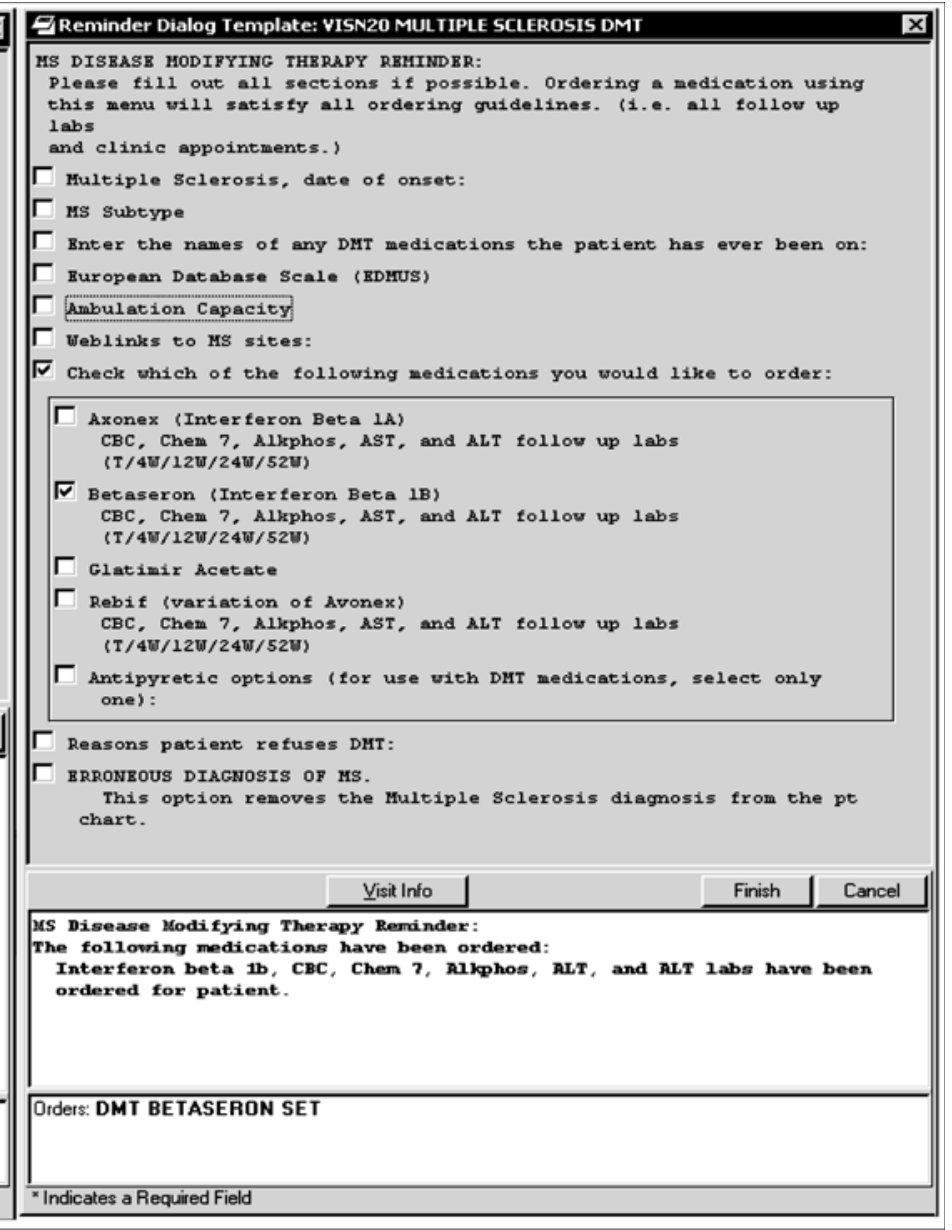

Figure 3.

Multiple sclerosis computerized clinical reminder dialog template. 
This is particularly important in MS, where the disease is progressive and has multiple associated secondary impairments, making the condition difficult to treat for the busy provider who does not care for large numbers of individuals with MS. Decision support can be accomplished without an informatics infrastructure; however, the use of enhanced EMRs coupled with supporting information on the World Wide Web can be an extremely powerful tool for affecting the decisions and behavior of clinical care providers. Such a system can promote evidence-based care across an entire healthcare system by informing providers and veterans about management strategies within the context of their daily care routines. These interventions can be embedded in the EMR, with links to supporting guidelines for those who seek additional information. The enhanced EMRs directly address the chronic care model goal of "promoting clinical care that is consistent with scientific evidence and patient preferences" [4]. Guidelines may enhance the EMR by creating disease-specific CCRs (Figure 3). CCRs promote evidence-based care for veterans with MS by suggesting appropriate interventions based on synthesized patient data (e.g., ICD codes, past interventions, laboratory results, and previous level of health) in underlying databases. This approach has been well evaluated for its role in improving rates of preventive health screening such as mammograms, vaccinations, and screening questionnaires [28-31]. CCRs have received less study for use in chronic care, but evaluations show that CCRs can help keep clinicians better "informed" and facilitate practice consistent with clinical practice guidelines in the management of chronic diseases such as diabetes [4-5,30-32].

To improve the impact and acceptance of CCR driven care, MSCoE developers are working to further enhance CPRS with embedded links to Web site reviews. These reviews provide further evidence or rationale to highlight the importance of the intervention suggested by a CCR. Much effort has been directed toward highly developed support of CCRs built around a guideline, since the presence of a CCR alone is not the sole determinant of success of decision-support tools. Explanation services, such as online help systems or, in this case, links to Web sites for supporting information, play a vital role in acceptance and impact of a decision-support tool [33]. When a provider is presented with the option to execute a CCR to assist him or her in the prescription of an MS DMA, a Web link can provide the necessary evidence and a logical, succinct description supporting the use of that treatment in individuals like the current patient and with a similar type of MS. The link can also provide prefilled prescription information, such as dosage, supporting laboratories, and follow-up visits.

Decision support is more effective when it is derived directly from individual patient data and the recommendations are based on individual patient characteristics [34]. The EMR (in our case, CPRS) is the meeting place for various types of data describing individual patient interactions with the clinical information system. The impact of a CCR-enhanced CPRS depends in part on how completely and accurately ICD codes, procedures, laboratory orders, medication orders, vaccinations, or problems lists are reflected in the underlying databases. As such, an effective CCR fills two roles: guiding the decisions made by healthcare providers and accurately capturing the outcomes of the use of a CCR in patient encounters. Proper encoding by the provider executing a CCR enhances the quality of underling data. If outcomes of the use of individual CCRs are not recorded effectively, then data describing the effectiveness of a given reminder or a given guideline will be unavailable or, worse, unreliable. Inaccurate or incomplete feedback or profiling reports will hurt user acceptance and will defeat the use of CCRs for quality assessment and QI. For example, a particular CCR may identify individuals by ICD code at high risk for influenza and pneumococcal pneumonia. A provider opening the chart of an individual in this cohort will be presented with the option to process a reminder to vaccinate. The patient will either get a vaccination or not. However, he or she may be allergic to the vaccine, so the provider elects not to vaccinate based on thoughtful deliberation. That this additional data be captured and the reminder turned off for the remainder of the risk period is important. Otherwise, another clinician gets the same reminder due to the absence of indication of vaccination. Profiling reports that assess only the presence or absence of an immunization lose valuable information. Clinicians' confidence in the entire reminder system can be diminished if problems like these are not addressed in the design and implementation of the reminder.

Given the complexity of care for individual with MS, the MSCoE has developed several sets of CCRs for the multiple aspects of MS care. One set of reminders pertains to the use of DMAs, with the goal of assuring that appropriate individuals with MS are offered treatment with a DMA. Many other routine aspects of MS care will be improved in the future by the use of CCRs, such as 
evaluation of neurogenic bladder and screening for depression and fatigue as well as vaccinations. Having reminders that not only effectively use existing data but also effectively capture new data will enhance both the EMR system and the richness and accuracy of the underlying MS data repository.

\section{TELEHEALTH DELIVERY OF SERVICES}

Telehealth involves the provision of healthcare and sharing of medical knowledge through telecommunications. Preventive, diagnostic, and therapeutic services, as well as patient education and assistance with selfmanagement of health, can be provided via telehealth. The potential for telehealth, particularly in the VHA, is quite dramatic, since veterans are often isolated by disability or geography. Research has shown that veterans tend to have more disabilities than the general population [35], and veterans can be especially challenged by geography in obtaining care. For example, Veterans Integrated Service Network (VISN) 20 serves approximately 160,000 veterans spread out over one-fifth of the landmass of the United States, covering 788,500 square miles with eight facilities.

Provision of care through the use of telehealth reduces the impact of disability caused by a chronic disease by providing care that does not depend on the ability of an individual to transport themselves to a medical facility. This includes care delivered by primary care providers or specialists for individuals in their home, consultation between primary care providers and specialists located miles away, or direct therapeutic treatment using one-on-one or group teletherapy. Without assistance, many disabled individuals only visit their providers when urgent care is required. For an individual to go through several hours of dressing, transfers, preparation of assistive mobility devices, and transportation for a $20 \mathrm{~min}$ educational session is not efficient. If a primary care provider needs advice on a specific management issue, specialist consultations require the patient to travel to the specialist. Through the use of telehealth, patients can visit with their providers more regularly for follow-up, counseling, or education. Primary-care physicians can meet with specialists with their patients present or not, simultaneously, or at different times. This ability to provide frequent, brief encounters aimed at education, improvement in self-management skills, and increased patient involvement in care can improve medical, emotional, and functional outcomes [36-44]. The ability to provide consultation services remotely reduces the barrier to receiving specialist consultation and will open the path to more frequent contact and better education of nonspecialists. In the language of the chronic-care model, telehealth provides a mechanism to "empower and prepare patients to manage their health and healthcare" while "assuring the delivery of effective, efficient clinical care and self-management support" [3-4].

For individuals with chronic and disabling diseases such as MS, providing "proactive care" and identification and treatment of "subpopulations for proactive care" [3-4] can be challenging. A successful chronic disease management approach relies on certain critical features such as "the provision of care in accord with an explicit plan, which includes regularly scheduled follow-up, systematic assessments and attention to the self-management needs of patients" [4]. These are the types of features that can be supported by a telehealth program (i.e., regularly scheduled communication; regular availability of data, photos, or other healthcare information; and a regularly monitored plan of care with self-management modules).

The population served by the MSCoE is a particularly appropriate target for telehealth. Based on a needsassessment study commissioned by the VHA in 2000, veterans with MS have significant barriers to care as a result of their disabilities [45]. The findings of this study indicate an overwhelming need for improved access. For example, 20 percent of patients surveyed reported that parking, distance, or transportation significantly interfered with receiving treatment at the VA. Half of those surveyed reported they had a severe gait disability or were mobile only with a wheelchair. A third of veterans surveyed required help getting to all activities and another third never drove. Yet only half the individuals surveyed reported they had a caregiver or family member to take them to the doctor. Twenty percent reported that they lived alone. Preliminary analyses suggest that restrictions in mobility are inversely associated with treatment of MS and secondary impairments. Without telehealth, disabling conditions may be limiting access to specialty centers and optimal treatment.

To address these limitations to access, the MSCoE is establishing a number of "virtual clinics." Telehealth clinics are divided into two categories, one-to-one communication (live) and asynchronous communication (storeand-forward). Live communication can include telephone 
contacts, simple videophone communication, or video communication enhanced by the ability to take photographs or vital signs during the visit. Store-and-forward communication does not require a live connection between provider and veteran. A veteran and/or caregiver logs into his or her telehealth device and, as requested, fills out a survey, measures vitals signs or takes a photograph. A provider reviews this information at a time that is convenient to his or her schedule or at a time when all other patients are reviewed. Vital signs are automatically loaded into the VHA's EMR system (in the VHA, the CPRS), and photographs and other information such as breath sounds or survey results are stored in an intermediary Web site. These telehealth contacts stretch the time between clinic visits by reducing the need for frequent follow-up appointments for an ongoing issue and by minimizing unplanned telephone calls or ER visits.

Telehealth has been described as a means to address the comprehensive needs of veterans with MS [46]. Within the MSCoE clinics, weekly telehealth visits have been established to provide check-in with individuals to discuss their ability to self-administer injectable medications, to follow up on medication changes such as those to treat spasticity or fatigue, or to address the concerns of individuals and their families. This regular telehealth clinic takes between 15 and 30 min and can support an individual with MS or their caregiver. Other telehealth devices are not synchronous, i.e., a scheduled visit is not needed. A device in an individual's home can be used to assess knowledge or adherence using a text-based interface. Blood pressure, skin photographs, and breath sounds can be stored and forwarded to a central location, attached to the EMR and reviewed when it is convenient for a clinician to do so. Abnormal values are flagged for priority evaluation.

Telehealth may also be used to connect nonspecialists such as primary-care doctors to specialists in more tertiary care centers. Multiple models for this type of interaction exist. Providers may meet on an educational basis for didactic or case-based education. Providers may also meet to discuss individual cases that represent diagnostic questions or the determination of whether a patient is suitable for referral. An example of a successful model in VISN 20 is the teleoncology tumor board that meets monthly. Providers sign in from throughout the VISN to present and discuss their individual patients and receive treatment advice and referral guidance.

Overall, the VHA is increasingly employing a wide range of telehealth approaches to many of its populations with chronic disease. These approaches include in-home monitoring of patient vitals signs and in-home communications via telephone or videophone, as well as the capability to photograph wounds or other patient characteristics and forward them to the EMR. The VA system also provides a robust network for interfacility telehealth communication. Telehealth activities supported by this network include administrative communication and employee education, as well as communication and consultation about care of individual patients. Interfacility telehealth consultations may be group case conferences, consultations forwarded as part of the EMR, or one-to-one communications from provider to provider.

Among other things, the MSCoEs are a model care system for organizational change within the entire VHA. To achieve widespread "delivery system redesign" and to "support improvement at all levels of the organization, beginning with senior leaders," an "MS Telehealth Toolkit" is being developed and will be distributed [3-4]. This toolkit is an electronic document that outlines the process other facilities are suggested to follow to replicate a telehealth program. This electronic document has many embedded forms and VA documents to assist the reader in filing appropriate applications, using standardized codes, and following commonly accepted evaluation techniques. This toolkit resides on the national VA telemedicine Web site and can be accessed by all VHA staff. The VHA telehealth initiative has created telehealth toolkits for various applications of telehealth. The intention is to take advantage of the "economy of scale," a medical system as big as the VHA. For example, Florida and Connecticut have developed the first home telehealth programs. These groups assembled a toolkit to be distributed to other medical centers wanting to start a telehome health program. The MS Telehealth Toolkit will be a vehicle to disseminate techniques for process redesign as well as "lessons learned" in the development of local telehealth programs. This will reduce the burden on individual medical centers to develop new telehealth programs from scratch.

\section{CONCLUSIONS}

The population-based, system-wide approach to management of individuals with chronic disease as described by Wagner et al. focuses on two related strategies: (1) educating and preparing clinical practice teams 
and (2) activating and educating individuals with chronic disease [3-4]. While Wagner's model is described independent of IT, the majority of actions called for in that model benefit tremendously from the application of a powerful informatics infrastructure designed to serve and support populations with chronic disease. This benefit is particularly true for a nationwide healthcare system like the VHA that shares common electronic medical tools, including a national EMR system, national data repositories, a well-developed Web infrastructure, and a developing cross-VHA telehealth plan. These interventions are also effective in other models of population-based care.

While an informatics infrastructure can greatly enhance care of populations of individuals with chronic conditions, numerous other conditions are necessary that do not depend on the effective use of IT. These include the creation of a culture and organization that promote safe, high-quality care; wise, active leadership; a systemwide openness to QI; and a reward system that promotes positive change.

Nevertheless, the MSCoEs are showing that, for the case of MS care, a well-designed and appropriately employed informatics infrastructure can strongly support the chronic-care model. QI efforts are greatly enhanced by the ability to analyze and disseminate outcomes and to monitor for errors. Consistency of care is increased by the ability to facilitate referral and coordination of health services within an organization and across multiple organizations. Individuals with chronic illness can better manage their health and healthcare through access to evidence-based information, interventions, and increased communication with care providers. These and other benefits can be obtained through the effective design and use of information and communication technologies in support of chronic-care management.

\section{ACKNOWLEDGMENT}

This material is the result of work supported with resources and the use of facilities at the Multiple Sclerosis Center of Excellence West, VHA, Seattle, Washington.

\section{REFERENCES}

1. Wagner EH, Grothaus LC, Sandhu N, Galvin MS, McGregor M, Artz K, Coleman EA. Chronic care clinics for dia- betes in primary care: A system-wide randomized trial. Diabetes Care. 2001;24(4):695-700. [PMID: 11315833]

2. Weiss KB, Wagner R. Performance measurement through audit, feedback, and profiling as tools for improving clinical care. Chest. 2000;118(2 Suppl):S53-58.

[PMID: 10940000]

3. Wagner EH. Chronic disease management: What will it take to improve care for chronic illness? Eff Clin Pract. 1998;1(1):2-4. [PMID: 10345255$]$

4. Wagner EH, Austin BT, Von Korff M. Organizing care for patients with chronic illness. Milbank Q. 1996;74(4):511-44. [PMID: 8941260]

5. Casalino L, Gillies RR, Shortell SM, Schmittdiel JA, Bodenheimer T, Robinson JC, Rundall T, Oswald N, Schauffler H, Wang MC. External incentives, information technology, and organized processes to improve health care quality for patients with chronic diseases. JAMA. 2003; 289(4):434-41. [PMID: 12533122$]$

6. Veterans Affairs Multiple Sclerosis Center of Excellence (MSCoE) [homepage on the Internet]. Seattle (WA): MSCoE; c1994-2006 [cited 2004 Aug 26]. About MSCoE; [1 screen]. Available from: http://www.va.gov/ms/about.asp

7. Cowper DC, Hynes DM, Kubal JD, Murphy PA. Using administrative databases for outcomes research: Select examples from VA Health Services Research and Development. J Med Syst. 1999;23(3):249-59. [PMID: 10554740]

8. Chernof B, Kaufman RL. Improving quality improvement: A data-driven assessment. West J Med. 1997;166(2):151-53. [PMID: 9109338]

9. Fry J. Ambulatory medical care data: Information for patient care in office-based practice. Med Care. 1973;11 (2 Suppl):35-40. [PMID: 4692410$]$

10. Institute of Medicine. Chronic care: Reducing the toll of chronic conditions on individuals and communities. In: Corrigan JM, Greiner A, Erickson SM, editors. Fostering rapid advances in health care: Learning from system demonstrations. Washington (DC): National Academy Press; 2003. p. 27-40.

11. Fry J. Record keeping in primary care. In: Sacket DL, Baskin MS, editors. Methods of health care evaluation. 2nd ed. Ontario (Canada): McMaster University; 1973.

12. Hamilton WT, Round AP, Sharp D, Peters TJ. The quality of record keeping in primary care: A comparison of computerised, paper and hybrid systems. Br J Gen Pract. 2003;53(497):929-33;discussion 933. [PMID: 14960216]

13. Rollman BL, Hanusa BH, Gilbert T, Lowe HJ, Kapoor WN, Schulberg HC. The electronic medical record. A randomized trial of its impact on primary care physicians' initial management of major depression [corrected]. Arch Intern Med. 2001;161(2):189-97. [PMID: 11176732] Erratum in: Arch Intern Med. 2001;161(5):705. 
14. McDonald WI, Compston A, Edan G, Goodkin D, Hartung H, Lublin FD, McFarland HF, Paty DW, Polman CH, Reingold SC, Sandberg-Wolheim M, Sibley W, Thompson A, Van den Noort S, Weinshenker BY, Wolinski JS. Recommended diagnostic criteria for multiple sclerosis: Guidelines from the International Panel on the diagnosis of multiple sclerosis. Ann Neurol. 2004;50(1):121-27. [PMID: 11456302]

15. Heller C. Leveraging information technology for quality improvement. Healthc Inf Manage. 1996;10(1):53-59. [PMID: 10155790]

16. McDonald CJ, Hui SL, Smith DM, Tierney WM, Cohen SJ, Weinberger M, McCabe GP. Reminders to physicians from an introspective computer medical record. A two-year randomized trial. Ann Intern Med. 1984;100(1):130-38. [PMID: 6691639]

17. Wilkinson EK, McColl A, Exworthy M, Roderick P, Smith $\mathrm{H}$, Moore M, Gabbay J. Reactions to the use of evidencebased performance indicators in primary care: A qualitative study. Qual Health Care. 2000;9(3):166-74. [PMID: 10980077]

18. Hackos JT, Redish JC. User and task analysis for interface design. Hoboken (NJ): Wiley; 1998.

19. Neilson J. Usability engineering. London (England): Academic Press; 1993.

20. Hunkeler EM, Meresman JF, Hargreaves WA, Fireman B, Berman WH, Kirsch AJ, Groebe J, Hurt SW, Braden P, Getzell M, Feigenbaum PA, Peng T, Salzer M. Efficacy of nurse telehealth care and peer support in augmenting treatment of depression in primary care. Arch Fam Med. 2000;9(8):700-708. [PMID: 10927707]

21. Baehring TU, Schulze H, Bornstein SR, Scherbaum WA. Using the World Wide Web-A new approach to risk identification of diabetes mellitus. Int J Med Inform. 1997; 46(1):31-39. [PMID: 9476153]

22. Lai C, Haselkorn JK, Martin C, Hatzakis M Jr, Haselkorn $\mathrm{M}$. The rest of the iceberg: Supporting an internal community of website content creators and reviewers. In: IEEE Professional Communicators Conference Proceedings; 2003.

23. Kirsch SE, Lewis FM. Using the World Wide Web in health-related intervention research. A review of controlled trials. Comput Inform Nurs. 2004;22(1):8-18. [PMID: 15069844]

24. Elson RB, Faughnan JG, Connelly DP. An industrial process view of information delivery to support clinical decision making: Implications for systems design and process measures. J Amer Med Inform Assoc. 1997;4(4):266-78. [PMID: 9223033]

25. Chueh HC, Barnett GO. "Just-in-time” clinical information. Acad Med. 1997;72(6):512-17. [PMID: 9200584]
26. Wilcox RA, Whitham EM. Reduction of medical error at the point-of-care using electronic clinical information delivery. Intern Med J. 2003;33(11):537-40. [PMID: 14656260]

27. Montori VM, Smith SA. Information systems in diabetes: In search of the holy grail in the era of evidence-based diabetes care. Exp Clin Endocrinol Diabetes. 2001;109 (Suppl 2): S358-72. [PMID: 11460584]

28. Tang PC, LaRosa MP, Newcomb C, Gorden SM. Measuring the effects of reminders for outpatient influenza immunizations at the point of clinical opportunity. J Amer Med Inform Assoc. 1999;6(2):115-21. [PMID: 10091064]

29. Cannon DS, Allen SN. A comparison of the effects of computer and manual reminders on compliance with a mental health clinical practice guideline. J Amer Med Inform Assoc. 2000;7(2):196-203. [PMID: 10730603]

30. Nilasena DS, Lincoln MJ. A computer-generated reminder system improves physician compliance with diabetes preventive care guidelines. Proc Annu Symp Comput Appl Med Care. 1995;640-45. [PMID: 8563365]

31. Nilasena DS, Lincoln MJ, Turner CW, Warner HR, Foerster VA, Williamson JW, Stults BM. Development and implementation of a computer-generated reminder system for diabetes preventive care. Proc Annu Symp Comput Appl Med Care. 1994;831-35. [PMID: 7950041]

32. Grant RW, Hamrick HE, Sullivan CM, Dubey AK, Chueh HC, Cagliero E, Meigs JB. Impact of population management with direct physician feedback on care of patients with type 2 diabetes. Diabetes Care. 2003;26(8):2275-80. [PMID: 12882848]

33. U.S. Preventative Services Task Force. Guide to clinical preventative services. 2nd ed. Washington (DC): U.S. Department of Health and Human Services, Office of Disease Prevention and Health Promotion; 1996.

34. Grimshaw JM, Russell IT. Effect of clinical guidelines on medical practice: A systematic review of rigorous evaluations. Lancet. 1993;342(8883):1317-22. [PMID: 7901634]

35. Kazis LE, Miller DR, Clark J, Skinner K, Lee A, Rogers W, Spiro A 3rd, Payne S, Fincke G, Selim A, Linzer M. Health-related quality of life in patients served by the Department of Veterans Affairs: Results from the Veterans Health Study. Arch Intern Med. 1998;158(6):626-32. [PMID: 9521227]

36. Cradock S. Helping patients to improve self management of diabetes. Heart. 2004;90(Suppl 4):iv 36-38; discussion iv 39-40. [PMID: 15145912]

37. Andersen BL. Biobehavioral outcomes following psychological interventions for cancer patients. J Consult Clin Psychol. 2002;70(3):590-610. [PMID: 12090371] Erratum in: J Consult Clin Psychol. 2003;71(3):481. 
38. Andersen BL. Psychological interventions for cancer patients to enhance the quality of life. J Consult Clin Psychol. 1992;60(4):552-68. [PMID: 1506503$]$

39. Bowles KH, Dansky KH. Teaching self-management of diabetes via telehomecare. Home Healthc Nurse. 2002; 20(1):36-42. [PMID: 11839963]

40. Murtagh J. Patient education. Chronic fatigue syndrome. Aust Fam Physician. 1995;24(7):1297. [PMID: 7661786]

41. DeLawter DE. Self-management of diabetes. Md Med J. 1991;40(9):815. [PMID: 1921661]

42. Goodall TA, Halford WK. Self-management of diabetes mellitus: A critical review. Health Psychol. 1991;10(1):1-8. [PMID: 2026125] Erratum in: Health Psychol. 1992; 11(1):77.

43. Mullen PD, Green LW, Persinger GS. Clinical trials of patient education for chronic conditions: A comparative meta-analysis of intervention types. Prev Med. 1985;14(6): 753-81. [PMID: 2418436]

44. Kelly CA, Barrett EJ, Nash J. Home monitoring and selfmanagement of diabetes in a rural population. Ir Med J. 1981;74(11):321-24. [PMID: 7309457]

45. Hatzakis M Jr, Turner AP, Williams RM, Bowen JD, Rodriquez AA, Haselkorn JK. Predictors of prescriptions for management of fatigue among veterans with multiple sclerosis. Arch Phys Med Rehabil. 2005;86(7):1376-80. [PMID: 16003667]

46. Hatzakis M Jr, Haselkorn JK, Williams RM, Turner AP, Nichol P. Telemedicine and the delivery of health services to veterans with multiple sclerosis. J Rehabil Res Dev. 2003;40(3):265-82. [PMID: 14582530]

Submitted for publication October 22, 2004. Accepted in revised form April 22, 2005. 